\title{
Effects of nematode infection on anorexia and leptin levels in lambs of two breeds*
}

\author{
K. Zaralis ${ }^{1,4}$, B.J. Tolkamp ${ }^{1}$, J.G.M. Houdijk ${ }^{1}$, A.R.G. Wylie ${ }^{2}$ \\ and I. Kyriazakis ${ }^{1,3}$ \\ ${ }^{1}$ Animal Nutrition and Health Department, Scottish Agricultural College, Kings Buildings \\ Edinburgh, EH9 3JG, United Kingdom \\ ${ }^{2}$ Agri-Food and Biosciences Institute, Newforge Lane, Queen's University of Belfast \\ Belfast BT9 5PX, Northen Ireland \\ ${ }^{3}$ Veterinary Faculty, University of Thessaly \\ P.O. Box 199, 43100 Karditsa, Greece
}

\begin{abstract}
The objective of this study was to investigate whether breed differences in production potential are associated with differences in the occurrence of anorexia following a nematode infection and whether plasma leptin concentrations elevate as a result of infection. Weaned lambs, of the Suffolk $\times$ Greyface cross and Scottish Blackface breed were either trickle infected with 21,000 infective Teladorsagia circumcincta larvae per week, or not infected. The results suggest that both the magnitude and duration of anorexia differ considerably between the two breeds and is likely to associate with their differences in immune response. In addition, results suggest that leptin has a role in the expression of immunity and parasite-induced anorexia in sheep.
\end{abstract}

KEY WORDS: anorexia, parasitism, leptin, circumcincta, breeds, sheep

\section{INTRODUCTION}

The recent literature suggests a link between the immune response and the occurrence of anorexia in parasitised ruminants. Greer et al. (2005) found that the anorexia that follows a primary infection with nematodes in young sheep can be completely abolished if an effective immune response is prevented. In addition, evidence from studies in mice and rats suggests that the adipocyte hormone leptin,

\footnotetext{
${ }^{1}$ Supported by SEERAD. Konstantinos Zaralis is grateful to the Hellenic State Scholarship Foundation for the provision of a postgraduate scholarship

${ }^{4}$ Corresponding author: e-mail: Konstantinos.Zaralis@sac.ac.uk
} 
plays a very important role in the regulation of immune responses. Leptin levels are acutely increased by inflammatory and administration of infectious stimuli (Faggioni et al., 2001). In intestinal inflammation in rats, elevated plasma leptin concentrations were correlated with the degree of inflammation, and associated with anorexia (Barbier et al., 1998). However, whether plasma leptin levels elevate during gastrointestinal nematode infections in sheep and whether it is associated with the parasite-induced anorexia is unknown.

In addition, a recently developed model postulates that a more resistant genotype is likely to exhibit lower magnitude and duration of anorexia compared to a genotype which is less resistant (Sandberg et al., 2006). However, although differences in resistance to nematode infections are likely to be associated with the production potential of the host (Coop and Kyriazakis, 1999), it is not known whether breeds differences in production potential are associated with differences in the degree of anorexia.

In this study, we test the hypotheses that during gastrointestinal nematode infections 1. lambs of a high growth potential will exhibit higher magnitude and longer duration of anorexia compared to lambs of a lower growth potential and 2. plasma leptin concentrations will increase as a result of nematode infection in growing lambs and will positively correlate with the degree of anorexia. Both hypotheses were tested during a primary and a secondary nematode infection.

\section{MATERIAL AND METHODS}

\section{Experiment 1}

Ninety-six weaned lambs, 48 Suffolk $\times$ Greyface crosses (S) and 48 Scottish Blackface (B), half male and half female were used. The lambs were approximately twelve weeks of age and were acclimatized for a period of three weeks prior to beginning experimental observations. Half of the lambs in each breed were infected (INF) with 21,000 Teladorsagia circumcincta larvae per week, while non-infected lambs were given a similar volume of water, thus undergoing the same amount of handling stress as the infected animals. A single diet (grass pellets) was used throughout the experiment. INF lambs were fed ad libitum while non infected lambs were fed either ad libitum $\left(\mathrm{C}_{\mathrm{al}} ; \mathrm{n}=12\right)$ or, restrictedly, at 90,80 or $70 \%$ of ad libitum. Treatments were balanced for breed, sex and initial lamb weight. The trial lasted for 12 weeks and lamb body weight, feed intake (FI) and faecal egg count (FEC) were recorded weekly. Blood samples were taken weekly and analysed for leptin concentrations using a homologous ovine-specific RIA developed at the Agri-Food and Biosciences Institute Laboratories, Belfast. 


\section{Experiment 2}

Forty-eight previously infected lambs, half S and half B were used. A period of two weeks intervened between the end of the Experiment 1 and the start of the Experiment 2 during which all lambs were fed ad libitum. At the start of the experiment all lambs were approximately 6 months of age. The experimental design, diets and facilities were identical to those used in Experiment 1, with the exception that only one level of feed restriction (90\% of ad libitum) was used in this Experiment. Measurements and procedures were carried out over similar time-scales and using identical protocols as described in Experiment 1.

\section{Statistical analysis}

Feed intake (FI), body weight (BW) and leptin data, were analysed by ANOVA using the MIXED procedure of SAS (SAS 9.1.3; SAS Institute Inc., Cary, NC). In all statistical models the repeated measurements over time were the experimental weeks. Data are reported as least square means and their standard error (SE) and their differences were tested by a t-test. Log-transformed FEC data were analysed by repeated measures ANOVA (GenStat Release 7.2 Lawes Agricultural Trust, Rothamsted Experimental Station).

\section{RESULTS}

Faecal samples taken ten days before the start of the primary infection indicated nematode eggs in the lambs' faeces. Thus, all lambs were immediately treated with anthelmintics before the start of the experiment. A significant breed difference $(\mathrm{P}=0.009)$ and a significant breed by time interaction $(\mathrm{P}=0.02)$ attributable the higher FEC of the $\mathrm{S}$ lambs compared to the $\mathrm{B}$ lambs observed during Experiment 1. In both breeds FEC reached a maximum value during the third week with a mean of $113(95 \%$ CI 81, 158) eggs/g and 73 (95\% CI 51, 102) eggs/g for the $\mathrm{S}$ and B lambs, respectively. From the third week of infection FEC declined gradually to zero values. FEC remained very low and did not differ between the two breeds $(\mathrm{P}=0.9)$ during Experiment 2.

The mean daily feed intake (FI) during the Experiment 1 is shown in Figure 1. Dosing with parasites caused a significant reduction in FI in $\mathrm{S}$ lambs $(\mathrm{P}<0.0001)$. The difference was significant after the second week $(\mathrm{P}=0.03)$ of the onset of infection and was consistent throughout the experiment (Figure 1). Mean daily FI of the INF $\mathrm{S}$ lambs was approximately $13 \%$ lower of that of $\mathrm{C}_{\mathrm{al}} \mathrm{S}$ lambs. Infection did not result in significant difference in FI between INF and $\mathrm{C}_{\mathrm{al}} \mathrm{B}$ lambs. 

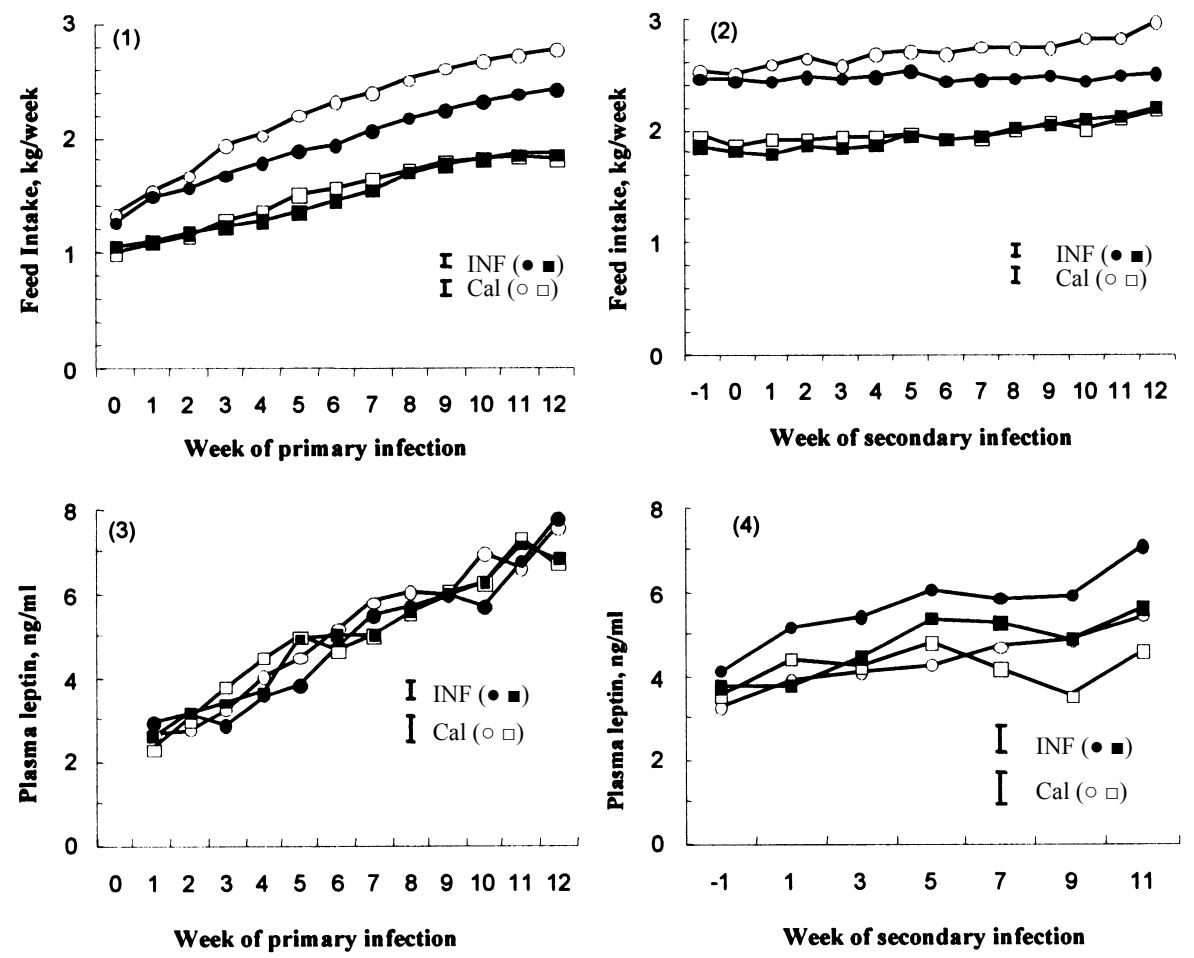

Figures 1-4. Daily average feed intake (Figures 1 and 2) and plasma leptin concentrations (Figures 3 and 4) in infected Suffolk (•), non-infected Suffolk (०), infected Blackface ( $\bullet$ ) and non-infected Blackface ( $\square$ ) fed ad libitum lambs, during the primary and the secondary infection. Average standard error of the treatments is also shown

The mean daily FI in the Experiment 2 is shown in Figure 2. The secondary infection caused a significant reduction in FI in INF2 $\mathrm{S}$ lambs, as indicated by the main effect of re-infection $(\mathrm{P}=0.015)$ and the interaction between infection and time $(\mathrm{P}=0.01)$. Daily FI in these lambs was approximately $10 \%$ lower than that of their $\mathrm{C} 2{ }_{\mathrm{al}}$ counterparts. No difference in FI was noted between INF2 and $\mathrm{C} 2{ }_{\mathrm{al}}$ B lambs $(\mathrm{P}=0.58)$.

In both breeds, leptin concentrations (PLC) increased significantly $(\mathrm{P}<0.001)$ with time and there was no significant interaction of breed and time $(\mathrm{P}=0.47)$. Feed restriction caused a significant reduction in $\mathrm{PLC}(\mathrm{P}=0.004)$. The effect of infection on $\mathrm{PLC}$ was not significant in either breed ( $\mathrm{P}>0.6$ ) during the Experiment 1 (Figure 3). Significantly higher PLC were observed during secondary infection in INF2 S lambs compared to $\mathrm{C} 2$ al $\mathrm{S}$ lambs $(\mathrm{P}=0.036)$ while no differences in $\mathrm{PLC}$ were noted between INF2 and $\mathrm{C} 2{ }_{\mathrm{al}} \mathrm{B}$ lambs (Figure 4). The differences in PLC between in INF2 and $\mathrm{C} 2{ }_{\mathrm{al}}$ $\mathrm{S}$ lambs were established after the first week of the secondary infection, while PLC in these groups did not differ prior the initiation of the secondary infection. 


\section{DISCUSSION}

The hypotheses tested in the experiments related to breed effects on lamb response to parasitic infection (in terms of FEC and FI), effects of infection on plasma leptin levels and the relationship between plasma leptin levels and anorexia in infected lambs.

The results showed that the two breeds differed in their ability to respond to gastrointestinal infections. Earlier studies also report that sheep selected for high productivity, have been observed to have high FEC and worm burden, compared to unselected animals (Amarante et al., 2004). Hence, the current study contributes and extends the body of evidence that resistance to infection is related with the production potential of the host (Coop and Kyriazakis, 1999). The relative low FEC observed herein is probably associated with the immune status of the lambs prior to the start of the trickle infection. The response of these lambs to the secondary infection seems to correlate with earlier findings in re-infected lambs (Seaton et al., 1989). However, the breeds used in the present study were likely to differ in their ability to express their acquired immunity to infection, if we consider the occurrence of anorexia and the high plasma leptin concentrations in the INF2 S lambs but not in the INF2 B.

The breed differences in resistance to infection were accompanied by differences in the magnitude and duration of anorexia. However, although breed differences in FEC were observed only during the primary infection, breed differences in the occurrence of anorexia were observed during both the primary and the secondary infection. These observations are consistent with the hypothesis tested that lambs of a high growth potential will exhibit higher magnitude and longer duration of anorexia compared to lambs of a lower growth potential. Moreover the results provided novel evidence that when the exposure to gastrointestinal nematodes in lambs is discontinued, a re-exposure to nematode infection can be associated with anorexia in lambs of a susceptible genotype to infection. The differences in anorexia between the two breeds are likely to be attributable to the differences in the immune responses developed in these lambs (Greer et al., 2005).

PLC can increase acutely during immune responses as evident by studies in mice and rats (Barbier et al., 1998). We therefore tested the hypothesis that nematode infection will result in an increased PLC in native lambs. The results presented here suggest a positive correlation between FI and PLC since restrictively fed lambs, had significantly lower PLC compared to the ad libitum fed lambs. In addition, the significant depression of appetite in the INF S lambs was not associated with a significant lower leptin level, which suggests that PLC remains high as a result of the infection. This suggestion seems plausible if we consider the outcome of the secondary infection. The results from Experiment 2 indicate clearly that mean plasma leptin concentrations in the INF2 S lambs 
were significantly higher compared to the mean leptin concentrations of the $\mathrm{C} 2$ al $\mathrm{S}$ lambs. Although there is limited evidence on the role of leptin on the immune response against gastrointestinal nematode infections in sheep, the outcome of the present study suggest a possible role of leptin in the regulation of immune responses and the occurrence of anorexia in parasitised lambs.

\section{CONCLUSIONS}

The results of these experiments provided evidence that differences in the magnitude and duration of anorexia exist between breeds that differ in production potential and that a secondary infection is likely to cause anorexia in growing lambs. Our findings suggest also that leptin has a possible role in the regulation of immune responses and the occurrence of anorexia in nematode parasitised animals.

\section{ACKNOWLEDGEMENTS}

The authors are grateful to the technical support teams of the Scottish Agricultural College and the Agri-Food and Biosciences Institute.

\section{REFERENCES}

Amarante A.F.T., Bricarello P.A., Rocha R.A., Gennari S.M., 2004. Resistance of Santa Ines, Suffolk and Ile de France sheep to naturally acquired gastrointestinal nematode infections. Vet. Parasitol. 120, 91-106

Barbier M., Cherbut C., Aube A.C., Blottiere H.M., Galmiche J.P., 1998. Elevated plasma leptin concentrations in early stages of experimental intestinal inflammation in rats. Gut 43, 783-790

Coop L.R., Kyriazakis I., 1999. Nutrition - Parasite interaction. Vet. Parasitol. 84, 187-204

Faggioni R., Feingold K., Grunfeld C., 2001. Leptin regulation of the immune response and the immunodeficiency of malnutrition. FASEB J. 15, 2565-2571

McAnulty R.W., Stankiewicz M., Sykes A.R., Greer A., 2005. Corticosteroid treatment prevents the reduction in food intake and growth in lambs infected with abomasal parasite Teladorsagia circumcincta. Proc. N.Z. Soc. Anim. Prod. 65, 9-12

Sandberg F.B., Emmans G.C., Kyriazakis I., 2006. A model for predicting feed intake of growing animals during exposure to pathogens. J. Anim. Sci. 84, 1552-1566

Seaton D.S., Jackson F., Smith W.D., Angus K.W., 1989. Development of immunity to incoming radiolabeled larvae in lambs continuously infected with Ostertagia-circumcincta. Res. Vet. Sci. 46, 241-246 